\title{
The electronic medical record in anesthesiology: a standard of quality healthcare and patient safety
}

\author{
Issam Tanoubi, MD, MA (Ed)
}

Received: 6 March 2017/Accepted: 12 April 2017/Published online: 17 April 2017

(C) Canadian Anesthesiologists' Society 2017

The electronic medical record (EMR) is increasingly being used to replace the paper anesthetic record in the operating room, and it is evident that it is not far from becoming a standard of care. The literature is rich in demonstrating its acceptability and ease of use, even if a certain time for adaptation is often necessary after its implementation. Furthermore, the use of the EMR as a clinical decision support tool is expected to enhance patient care and safety, which is also increasingly being highlighted in the current literature. Indeed, in this issue of the Journal, Hincker et al. show how improvements in compliance with guidelines for repeat cefazolin administration in the perioperative period can be made by adding inexpensive nonintrusive interventions to the EMR. ${ }^{1}$ In their before-and-after cohort analysis, they showed that this was achieved through the use of a simple bar on the EMR monitor that displayed continuously from the start of the initial antibiotic dosing and ended $15 \mathrm{~min}$ before subsequent doses were indicated. Such a straightforward adjustment to the EMR management software can have a direct effect on a practitioner's adherence to practice guidelines and could ultimately impact patient safety. Importantly, however, there are other areas in the EMR besides those related to antibiotic administration that could also be modified to improve patient safety. ${ }^{2}$

\footnotetext{
I. Tanoubi, MD, MA (Ed) ( $\square)$

Department of Anesthesiology, Centre d'Apprentissage des Attitudes et Habiletés Cliniques (CAAHC), Université de Montréal, Pavillon Roger-Gaudry, 2900, boul. ÉdouardMontpetit, Montreal, QC, Canada

e-mail: i.tanoubi@umontreal.ca
}

\section{Preventing medical errors}

During anesthetic management, perioperative events are often repeated at regular intervals (e.g., tranexamic acid or ketamine administration) or there may be the need to administer drugs in particular clinical situations, such as calcium during blood transfusion or additional antiemetic agents for use when patients are at particularly high risk of postoperative nausea and vomiting. Reminders to respond to these events can easily be integrated into the EMR, either in the form of a time bar for repeatedly administered drugs, such as the intervention evaluated by Hincker et al., ${ }^{1}$ or in the form of an "if event $X$ is present then send a recall to do $Y$ " loop. These reminders are all the more important as the events that they prompt can occur during intraoperative moments of high cognitive workload (e.g., during patient hemorrhage).

With these types of reminders, the EMR can continue serving as a support tool for clinical decision-making and, without being excessively intrusive, allow the practitioner to adhere to practice guidelines. This contrasts with an intrusive intervention, such as a high-decibel alarm that can be easily confused with a different alarm, or a visual signal that occupies the entire screen and can hide patient-related parameters. These interventions could have negative effects-i.e., they could generate a disturbing cognitive overload or lead to alert desensitization, thus decreasing their effectiveness.

Attention is a valuable resource to preserve; it can often be limited and easily overloaded and exhausted. Many clinicians already use everyday electronic devices as aids (e.g., smartphone reminder alerts) to address certain challenges to their attention, particularly for various types of reminders in their routine daily life. It only makes sense for practitioners to use the EMR similarly for reminders of 
important intraoperative events and tasks. In doing so, the EMR can help prevent medical errors that might occur due to inattentiveness or omission associated with intrinsic or extrinsic cognitive overload. Undesirable events, such as adverse drug administration or drug interactions, could thus be avoided.

There is also potential for error when we must rely on the accuracy of handwritten documentation in the intraoperative medical record. For example, we can appreciate that handwritten notes, which often include syntax errors or non-standardized acronyms, could be difficult for others to understand and could lead to loss of information or confusion when transferred between healthcare providers. Indeed, patient mishaps are known to develop through a combination of several factors (or failures), including the failure to transmit accurate information between healthcare providers. ${ }^{3}$ The accuracy and completeness of clinical documentation have improved through the use of the EMR. Improvements in the information presentation functions within the EMR have reduced ineffective cognitive load resulting from the need to search for relevant information, refine the information, and decipher handwritten notes. Thanks to the EMR, this ineffective cognitive load, which can occupy a considerable space in our working memory, can thus be reduced and replaced with a higher level of accurate and relevant information that is ready for use. ${ }^{4}$

\section{Crisis resource management and the EMR}

A medical crisis is defined as a situation where the available resources are less than those required to treat the patient adequately - and such resources can include cognitive capacity. The practitioner is not immune to forgetfulness as regards treatment algorithms, the correct frequency of necessary drug doses, and the steps and tasks required to optimize patient care. In this context, an important tool to assist with crisis management is the use of various cognitive aids. Several cognitive aids and algorithms, such as critical event and crisis checklists (with a printed "pamphlet" or poster or a mobile application), are currently available at the anesthesia work station in some hospitals. Paradoxically, trainees frequently do not use these aids during simulation, in part because they don't know where the aids are located or because the aids are not user friendly. In addition, investigations into the use of cognitive aids during simulated crises have drawn differing conclusions. ${ }^{5}$ Finding the best cognitive aid design is a continuing goal. For the EMR, one can imagine clicking on a "rare situation icon" on the EMR screen. Once the icon is clicked, the encountered problem would be displayed and treatment algorithms would be visualized and incorporated in a step-by-step manner, with drugs adapted to the patient's weight and age. Clicking this type of aid on the EMR screen could also include links to other reminders of crisis resource management, including the distribution and prioritization of tasks and communications and/or reminders to call for help when needed. The use of both passive (e.g., guidelines) and active (e.g., specific management steps) aids to modify a clinician's behaviour are expected to enhance patient health and safety.

\section{Data collection}

There have been recent innovative trends regarding the use of data sources for analyses in healthcare. From a research perspective, the EMR offers significant opportunities for data analyses. Current EMR systems facilitate rapid accumulation of patient data in an electronic format, allowing hospital organizations to assess quality of care more reliably. Through analyses of data gathered through the EMR, operating room organization has also improved in such areas as managing resources and conducting risk assessments. ${ }^{6}$

Clearly, concise patient data analyses would not be possible with paper medical records that may have missing, unreliable, and/or inaccurate data. ${ }^{7}$ For example, trying to associate intraoperative events, e.g., hypotension, with postoperative mobility or mortality is difficult as these events are often poorly documented. Accordingly, there would be uncertainty as to how results of such analyses would have a positive impact on patient safety. More troublesome, results could impact patient safety by showing no association between low mean arterial pressure and intraoperative morbidity and the overall outcome of the patient.

The EMR has also facilitated uncovering the poor association between blood pressure management by anesthesia residents and competence committee evaluations or in-training exam performance. ${ }^{8}$ For example, in a retrospective cohort analysis of 1,807 hypotension episodes, which were obtained from the EMRs of 10,065 operations managed by 70 first- and second-year anesthesia residents at the Cleveland Clinic, Sessler et al. investigated whether the duration of severe hypotension during anesthesia administered by residents correlates with performance evaluations and in-training exam scores. The results of their analysis showed no association between the characteristics of blood pressure management and evaluations of clinical competence. Even if criticism persists on certain aspects of these findings (such as the validity of this new measure as a tool for assessing resident competence), it raises (at least for the 
trainers) the issue of the accuracy of our practice assessment methods. Perhaps relevant data collected from the anesthesia EMR would reflect a trainee's skills better than several in-training competence assessments. ${ }^{9}$ It is easy to fathom how improving future anesthesiologists' skills and performance could have an impact on patient safety, although other factors may interfere with this process.

Overall, use of the EMR system as a decision support tool for anesthesiologists seems straightforward-i.e., search for the source of a recurring error, create an automatic alert, and observe the effect of this intervention on practitioners' behaviour (and ultimately on patient safety). ${ }^{10}$ Some barriers, usually organizational or related to procedural change, still slow widespread use of EMRs. ${ }^{11}$ Nevertheless, it remains clear that adopting this approach will facilitate communication, patient care, and research, and ultimately lead to enhanced patient safety.

\section{Le dossier médical informatisé en anesthésiologie : une norme pour des soins de santé de qualité et la sécurité des patients}

Le dossier médical informatisé (DMI) (ou dossier médical électronique (DME), comme il est parfois appelé) est de plus en plus utilisé pour remplacer le dossier anesthésique en format papier en salle d'opération, et il est évident qu'il est en passe de devenir la norme de soins. La littérature démontrant son acceptabilité et sa facilité d'utilisation est abondante, même si une période d'adaptation est souvent nécessaire après sa mise en œuvre. En outre, on prévoit que l'utilisation d'un DMI en tant qu'outil de soutien à la décision clinique améliorera les soins et la sécurité des patients, un atout que la littérature actuelle souligne de plus en plus. En effet, dans ce numéro du Journal, Hincker et coll. montrent dans quelle mesure le respect des directives concernant les doses de rappel de céfazoline en période périopératoire peut être amélioré en ajoutant des interventions peu dispendieuses et non intrusives au DMI. ${ }^{1}$ Dans leur analyse de cohorte avant-après, les auteurs ont montré que ce résultat avait été obtenu en ajoutant une simple barre au moniteur de DMI, affichée en continu dès le moment de la première administration d'antibiotiques et jusqu'à 15 minutes avant que l'indication pour des doses subséquentes n'apparaisse. Ce simple ajustement au logiciel de gestion du DMI peut avoir un effet direct sur le respect, par un praticien, des directives de pratique, et pourrait avoir un impact sur la sécurité des patients en bout de ligne. Il est toutefois important de souligner que d'autres éléments du DMI, outre ceux liés à l'administration d'antibiotiques, pourraient également être modifiés pour améliorer la sécurité des patients. ${ }^{2}$

\section{Prévention des erreurs médicales}

Pendant la prise en charge anesthésique, plusieurs actes périopératoires sont souvent répétés à intervalles réguliers (par ex., l'administration d'acide tranexamique ou de kétamine); certains médicaments pourraient devoir être administrés dans certaines situations cliniques particulières, comme par exemple du calcium pendant les transfusions sanguines ou des agents antiémétiques supplémentaires lorsque les patients courent un risque particulièrement élevé de nausées et vomissements postopératoires. Il serait très facile d'intégrer des rappels pour de tels événements dans le DMI, que ce soit sous forme de barre temporelle pour les médicaments administrés à répétition, comme dans l'intervention évaluée par Hincker et coll., ${ }^{1}$ ou sous forme de boucle de type « si l'événement $X$ est présent, alors afficher un rappel pour faire $Y »$. Ces rappels sont d'autant plus importants étant donné que les complications qu'ils provoquent peuvent survenir pendant des périodes peropératoires durant lesquelles la charge de travail cognitif est élevée (par ex., pendant une hémorragie).

Avec ce type de rappels, le DMI peut continuer à servir d'outil d'aide à la prise de décision clinique et, sans être excessivement intrusif, permettre au praticien de respecter les directives de pratique. Ces rappels sont en contraste avec une intervention intrusive, comme par exemple une alarme très forte, qui pourrait facilement être confondue avec une autre alarme, ou un signe visuel qui occuperait l'écran entier et cacherait les paramètres liés au patient. De telles interventions pourraient avoir des effets négatifs : ils pourraient par exemple entraîner une surcharge cognitive dérangeante ou mener à une désensibilisation aux alertes, réduisant ainsi d'autant plus leur efficacité.

L'attention est une ressource précieuse qu'il est important de préserver; elle est souvent limitée et peut facilement être surchargée ou épuisée. Bon nombre de cliniciens utilisent déjà des dispositifs électroniques de tous les jours pour les aider (par ex., des rappels sur leur téléphone intelligent) à répondre à certains défis posés à leur attention, particulièrement en ce qui touche divers types de rappels dans leur vie quotidienne. Ainsi, il est tout à fait logique que les praticiens utilisent le DMI de la même façon - pour obtenir des rappels des événements et tâches peropératoires importants. Ce faisant, le DMI peut contribuer à prévenir les erreurs médicales qui pourraient survenir en raison d'un moment d'inattention ou d'une omission associée à une surcharge cognitive intrinsèque ou 
extrinsèque. Des événements indésirables, tels qu'une administration médicamenteuse ou des interactions médicamenteuses néfastes, pourraient ainsi être évités.

Le potentiel de commettre une erreur existe aussi lorsqu'on n'a d'autre choix que de se fier à l'exactitude d'une documentation écrite à la main dans un dossier médical peropératoire. Par exemple, nous sommes tous conscients que des notes écrites à la main, qui incluent souvent leur lot d'erreurs syntaxiques et d'acronymes non standardisés, peuvent être difficiles à comprendre et pourraient mener à une perte d'informations ou à confusion lorsque ces informations sont transférées entre divers professionnels de la santé. En effet, nous savons que les incidents dont sont victimes les patients sont souvent dus à une combinaison de plusieurs facteurs (ou échecs), y compris l'échec de transmission d'informations précises entre professionnels de la santé. ${ }^{3} \mathrm{La}$ précision et l'exhaustivité de la documentation clinique se sont améliorées depuis l'implantation du DMI. Les améliorations apportées aux fonctions de présentation des informations au sein du DMI ont permis de réduire la charge cognitive inefficace résultant de la nécessité de chercher les informations pertinentes, de valoriser ces informations et de déchiffrer des notes écrites à la main. Grâce au DMI, cette charge cognitive inefficace, qui peut occuper un espace considérable dans notre mémoire à court terme, peut ainsi être réduite et remplacée par un niveau plus élevé d'informations précises, pertinentes et prêtes à l'emploi. ${ }^{4}$

\section{Gestion des ressources en cas de crise et DMI}

On définit une crise médicale comme une situation dans laquelle les ressources disponibles sont moins importantes que celles nécessaires à traiter le patient de façon appropriée - et la capacité cognitive peut faire partie de telles ressources. Le praticien n'est pas à l'abri des pertes de mémoire en ce qui touche aux algorithmes de traitement, à la fréquence correcte d'administration de médicaments nécessaires, et aux étapes et tâches nécessaires pour maximiser les soins au patient. Dans un tel contexte, un outil important pour aider à la gestion de crise est l'utilisation de diverses aides cognitives/listes de vérification. Plusieurs aides cognitives et algorithmes, tels que les listes de vérification en cas d'événements critiques et de crise (sous forme de pamphlet imprimé, d'affiche ou d'application mobile), sont actuellement disponibles au poste de travail de l'anesthésie dans certains hôpitaux. Paradoxalement, il est encore très fréquent que les stagiaires n'utilisent pas ces aides pendant les simulations, soit parce qu'ils ne savent pas où elles se trouvent, ou parce que ces aides ne sont pas conviviales. En outre, les recherches sur l'utilisation des aides cognitives pendant des crises simulées sont parvenues à des conclusions divergentes. ${ }^{5}$ Nous sommes toujours à la recherche du meilleur concept d'aide cognitive. En ce qui a trait au DMI, on peut imaginer cliquer sur une « icône de situation rare » sur l'écran. Une fois qu'on a cliqué sur l'icône, le problème rencontré pourrait être affiché, les algorithmes de traitement visualisés et intégrés de façon progressive ou étape par étape, et les médicaments pourraient être adaptés en fonction du poids et de l'âge du patient. En cliquant sur ce type d'aide sur l'écran du DMI, on pourrait également avoir accès à des liens vers d'autres rappels de gestion des ressources en cas de crise, notamment la distribution et la priorisation des tâches et des communications et/ou des rappels pour demander de l'aide si nécessaire. L'utilisation d'aides passives (par ex. des directives) et actives (par ex. des étapes de prise en charge spécifiques) pour modifier le comportement d'un clinicien devrait nous permettre d'améliorer la santé et la sécurité des patients.

\section{Collecte de données}

Récemment, plusieurs tendances innovantes sont apparues quant à l'utilisation des sources de données pour réaliser des analyses dans les soins de santé. Du point de vue de la recherche, le DMI offre d'importantes possibilités d'analyse de données. Les systèmes de DMI actuels facilitent l'accumulation rapide de données de patients dans un format informatisé, ce qui permet aux organismes hospitaliers d'évaluer la qualité des soins prodigués de façon beaucoup plus fiable qu'auparavant. Grâce à l'analyse de données colligées via le DMI, l'organisation de la salle d'opération a également vu des améliorations dans des domaines tels que la gestion des ressources et l'évaluation des risques. ${ }^{6}$

Il est évident que des analyses concises des données de patients seraient impossibles avec des dossiers médicaux en format papier, où certaines données pourraient manquer, être peu fiables et/ou imprécises. ${ }^{7}$ Par exemple, il est difficile d'associer des événements peropératoires, comme l'hypotension, à une mobilité ou une mortalité postopératoires, étant donné que ces événements sont bien souvent mal documentés. De la même manière, nous ne pourrions pas savoir la mesure dans laquelle les résultats de telles analyses pourraient avoir un impact positif sur la sécurité des patients. Ce qui est plus troublant, c'est que ces résultats pourraient influencer la sécurité des patients en ne montrant aucune association entre une tension artérielle moyenne basse et une morbidité peropératoire et le pronostic global du patient.

Le DMI a également facilité la mise en évidence l'absence de corrélation entre la prise en charge de la 
tension artérielle par les résidents en anesthésie et les évaluations des compétences par un comité ou la performance aux examens en cours de formation. ${ }^{8} \mathrm{Par}$ exemple, dans une analyse de cohorte rétrospective de 1807 épisodes d'hypotension, tirés des DMI de 10065 opérations prises en charge par 70 résidents en anesthésie de première et deuxième année à la Clinique de Cleveland, Sessler et coll. ont tenté de déterminer si la durée d'hypotension grave pendant une anesthésie administrée par les résidents était corrélée aux évaluations de performance et aux notes d'examens de ces résidents en cours de formation. Les résultats de leur analyse n'ont pas montré d'association entre les caractéristiques de la prise en charge de la tension artérielle et les évaluations de compétence clinique. Même si la critique persiste concernant certains aspects de ces résultats (tels que la validité de cette nouvelle mesure en tant qu'outil d'évaluation de la compétence des résidents), cela soulève (du moins pour le personnel enseignant) la question de la précision de nos méthodes d'évaluation de la pratique. Peut-être que des données pertinentes, colligées à partir des DMI d'anesthésie, reflèteraient les compétences d'un stagiaire mieux que plusieurs évaluations de la compétence en cours de formation. ${ }^{9}$ Il est facile de voir comment l'amélioration des compétences et de la performance des futurs anesthésiologistes pourrait avoir un impact sur la sécurité des patients, bien que plusieurs autres facteurs influencent sans doute aussi ce processus.

Globalement, l'utilisation du système de DMI en tant qu'outil d'aide à la décision pour les anesthésiologistes semble simple - c'est-à-dire, chercher la source d'une erreur récurrente, créer une alerte automatique pour y pallier et observer l'effet de cette intervention sur le comportement des praticiens (et donc sur la sécurité des patients). ${ }^{10}$ Certains obstacles, habituellement de nature organisationnelle ou liés au changement de procédure, ralentissent encore l'utilisation répandue des DMI. ${ }^{11} \mathrm{Il}$ demeure cependant clair que l'adoption de cette approche facilitera la communication, les soins aux patients, la recherche, et mènera en définitive à une meilleure sécurité des patients.
Conflicts of interest None declared.

Editor responsibility This submission was handled by Dr. Hilary P. Grocott, Editor-in-Chief, Canadian Journal of Anesthesia.

Conflit d'intérêt Aucun.

Responsable de l'éditeur Cet article a été traité par Dr Hilary P. Grocott, rédacteur en chef, Journal canadien d'anesthésie.

\section{References}

1. Hincker A, Ben Abdallah A, Avidan M, Candelario P, Helsten D. Electronic medical record interventions and recurrent perioperative antibiotic administration: a before-and-after study. Can J Anesth 2017; 64: this issue.

2. Smith SK, de Lisle Dear G, Cooper SH, Taicher BM. Electronic reminders for intraoperative antibiotic re-dosing. Can J Anesth 2017. DOI:10.1007/s12630-017-0825-0.

3. Reason J. James Reason: patient safety, human error, and Swiss cheese. Interview by Karolina Peltomaa and Duncan Neuhauser. Qual Manag Health Care 2012; 21: 59-63.

4. Young JQ, Van Merrienboer J, Durning S, Ten Cate O. Cognitive Load Theory: implications for medical education: AMEE Guide No. 86. Med Teach 2014; 36: 371-84.

5. Marshall S. The use of cognitive aids during emergencies in anesthesia: a review of the literature. Anesth Analg 2013; 117 : 1162-71.

6. Rudolph JL, Doherty K, Kelly B, Driver JA, Archambault E. Validation of a delirium risk assessment using electronic medical record information. J Am Med Dir Assoc 2016; 17: 244-8.

7. Jang J, Yu SH, Kim CB, Moon Y, Kim S. The effects of an electronic medical record on the completeness of documentation in the anesthesia record. Int J Med Inform 2013; 82: 702-7.

8. Sessler DI, Makarova N, Riveros-Perez, R, Brown DL, Kimatian $S$. Lack of association between blood pressure management by anesthesia residents and competence committee evaluations or intraining exam performance: a cohort analysis. Anesthesiology 2016; 124: 473-82.

9. Weller J. What can electronic anesthesia records tell us about resident competence? Anesthesiology 2016; 124: 259-60.

10. Simpao AF, Galvez JA, Cannesson M. Should we fear computers or the lack of them? Technology, digital quality improvement, and the care redesign process. Anesthesiology 2017; 126: 369-70.

11. Boonstra A, Broekhuis M. Barriers to the acceptance of electronic medical records by physicians from systematic review to taxonomy and interventions. BMC Health Serv Res 2010; 10: 231. 